\title{
Identification and Regulation of Reticulon 4B (Nogo-B) in Renal Tubular Epithelial Cells
}

\author{
Ethan P. Marin, ${ }^{*}$ Gilbert Moeckel, ${ }^{\dagger}$ \\ Rafia Al-Lamki, ${ }^{\ddagger}$ John Bradley, ${ }^{\ddagger}$ Qingshang Yan, ${ }^{\S}$ \\ Tong Wang, ${ }^{\S}$ Paulette L. Wright, ${ }^{\text {TाI }}$ Jun Yu, ${ }^{\text {,III }}$ \\ and William C. Sessa ${ }^{\text {TIII }}$

\begin{abstract}
From the Departments of Nephrology, ${ }^{*}$ Pathology, ${ }^{\dagger}$ Physiology, ${ }^{\S}$ Pharmacology," and the Vascular Biology and Therapeutics of Health Research Cambridge Biomedical Research Centre, Addenbrooke's Hospital, University of Cambridge, Cambridge, United Kingdom
\end{abstract} \\ Program," Yale University School of Medicine, New Haven, \\ Connecticut; and the Department of Medicine, ${ }^{\ddagger}$ National Institute
}

Nogo-B is a member of the reticulon family of proteins that has been implicated in diverse forms of vascular injury. Although Nogo-B is expressed in renal tissues, its localization and function in the kidney have not been examined. Here, we report that Nogo-B is expressed specifically in the epithelial cells of the distal nephron segments in the murine kidney. After unilateral ureteral obstruction (UUO) and ischemia/reperfusion, Nogo-B gene and protein levels increased dramatically in the kidney. This increase was driven in part by injury-induced de novo expression in proximal tubules. Examination of Nogo-B immunostaining in human biopsy specimens from patients with acute tubular necrosis showed similar increases in Nogo-B in cortical tubules. Mice genetically deficient in Nogo-A/B were indistinguishable from wild-type (WT) mice based on histological appearance and serum analyses. After UUO, there was a significant delay in recruitment of macrophages to the kidney in the Nogo-A/B-deficient mice. However, measurements of fibrosis, inflammatory gene expression, and histological damage were not significantly different from WT mice. Thus, Nogo-B is highly expressed in murine kidneys in response to experimental injuries and may serve as a marker of diverse forms of renal injury in tissues from mice and humans. Furthermore, Nogo-B may regulate macrophage recruitment after UUO, although it does not greatly affect the degree of tissue injury or fibrosis in this model. (Am J Pathol 2010, 177:2765-2773; DOI: 10.2353/ajpath.2010.100199)

Reticulons (Rtn; Rtn 1-4) are proteins involved in formation of the reticular endoplasmic reticulum ${ }^{1,2}$ and may exert additional functions in regulating protein transport, ${ }^{3,4}$ signaling, ${ }^{5,6}$ and cell survival. ${ }^{7}$ In the Rtn 4 family, there are three isoforms termed Nogo-A, -B, and -C. Nogo-A is predominantly expressed in myelinated nerves within the CNS, where it is a component of a myelin inhibitory complex that retards axonal outgrowth. ${ }^{8,9}$ Nogo-C is expressed mainly in skeletal muscle and within the CNS, and its function is not well understood. Nogo-B is highly expressed in many cells in culture and primarily found in blood vessels and heart in vivo. Previous work from our group has shown that Nogo-B serves a negative regulator of vascular injury ${ }^{5}$ and possibly regulates the extent of injury of other tissues as well.

Mice genetically deficient in both Nogo-A/B (Nogo-A/B $\mathrm{KO})$ demonstrate excessive neointimal hyperplasia after arterial wire injury, an effect that can be reversed by adenoviral mediated gene transfer of Nogo-B. ${ }^{5}$ In both normal mice and in a pig model of vein graft remodeling, gene transfer of Nogo-B blocks excessive neointimal formation. ${ }^{10} \mathrm{~A}$ role for Nogo in regulating cardiovascular function in humans has been suggested by independent laboratories showing the following: (A) Nogo protein levels in serum were elevated in heart failure ${ }^{11}$; (B) Nogo gene expression levels were reduced in cardiac tissue of humans with heart failure as well as in mice with experimental heart failure ${ }^{12}$; (C) Nogo-B protein levels were reduced in human atherosclerotic plaques ${ }^{13,14}$; and (D) Nogo-B protein and gene levels were reduced in human aortic aneurysms. ${ }^{15}$ How Nogo-B affects these processes are unknown, but there is evidence supporting both an intracellular and intercellular role for this interesting protein.

The role of reticulons in the kidney is not known. Nogo-B (but not Nogo-A or Nogo-C), ${ }^{16}$ as well as Nogo receptors ${ }^{6,17}$ are found within the kidney. Here we show that Nogo-B is expressed primarily in epithelial cells of the distal nephron segments in the murine kidney. Nogo

Supported by grants R01 HL64793, R01 HL61371, R01 HL081190, R01 HL 096670, and P01 HI70295 from the National Institutes of Health (W.C.S.), a National Kidney Foundation Postdoctoral Research Fellowship (E.P.M.), a Scientist Development grant (0930157N) from the American Heart Association (J.Y.), and the Cambridge Biomedical Research Centre and British Heart Foundation (R.A.-L. and J.B.).

Accepted for publication August 19, 2010.

Address reprint requests to William C. Sessa, Ph.D., Yale University School of Medicine, New Haven CT 06536-0812. E-mail: william.sessa@yale.edu. 
expression is significantly increased after experimental injuries in mice, as well as in acute tubular necrosis (ATN) in humans. Nogo-A/B KO mice are indistinguishable from wild-type (WT) mice at baseline. However, after unilateral ureteral obstruction (UUO), macrophage recruitment is significantly delayed in Nogo-A/B KO mice relative to WT. This delay was not correlated with statistically significant reductions in renal injury as judged by histological assessments. In sum, Nogo-B is a novel marker of diverse renal injuries and a regulator of macrophage recruitment to the obstructed kidney. However, it does not significantly determine the course of UUO as performed in this study.

\section{Materials and Methods}

\section{Reagents}

Chemicals were obtained from Sigma-Aldrich unless otherwise noted. Anti-Nogo antiserum was raised in rabbits using a peptide derived from the amino terminus of Nogo-A/B and was used in all experiments to detect Nogo in tissue or in Western blots, except for analysis of human biopsy specimens, where $\alpha$-Nogo N-18 antibody (Santa Cruz Biotechnology, Santa Cruz, CA) was used. Other reagents used were anti-aquaporin-2 antibody C-17 (Santa Cruz Biotechnology); anti- $\beta$ COP (ABR - Affinity Bioreagents); anti-F4/80 (Caltag); fluorescein-labeled dolichos biflorus agglutinin lectin (DBA; Vector Laboratories, Burlingame, CA); and fluorescein-labeled lotus tetragonolobus lectin (LTL; Vector Laboratories).

\section{Mouse Handling and Experimental Injuries}

All procedures for handling and experimenting with mice were approved by the Institutional Animal Use \& Care Committee of Yale University. Nogo-A/B KO mice ${ }^{18}$ were a gift from Dr. Tessier-Lavigne (Genentech, South San Francisco, CA). They were bred to be congenic to C57BL/6 mice (confirmed by genotyping at the University of Missouri Research Animal Diagnostic Laboratory), which were used as WT mice. Nogo lacZ reporter mice, ${ }^{19}$ which were also deficient in expression of Nogo-A/B, were a gift from Dr. Strittmatter (Yale). Mice were housed in standard alternating 12-hour light/dark conditions and given free access to water and mouse chow. They were studied at 8-12 weeks of age. Mice were given ketamine/ xylazine anesthesia for all surgical procedures. For unilateral ureteral obstruction, the ureter was exposed via a small flank incision. The ureter was obstructed with two sequential sutures placed as close to the hilum as possible. For ischemia/reperfusion, the renal vasculature was approached via a central abdominal incision. The entire left renal pedicle was occluded with a vascular clamp for 30 minutes; the opposite artery was exposed but not clamped. In both surgeries, the skin was closed with surgical staples, and the deeper tissue layers were closed with sutures. Animals were given buprenorphine SQ $(0.05 \mathrm{mg} / \mathrm{kg}) \mathrm{q} 12$ hours for 48 hours postop. Mice were sacrificed at appropriate time by injection with ket- amine and Xylazine. Plasma samples were collected and stored at $-80^{\circ} \mathrm{C}$. Subsequently, the mice were perfused with phosphate buffered saline (PBS) via the left ventricle. Kidneys were collected, washed, decapsulated, and then processed as needed for histology and/or protein and RNA extraction. Serum creatinine (SCr) was determined by liquid chromatography at the Mouse Metabolic Phenotyping Center at Yale University. Urine osmolality was determined in the Yale-New Haven Hospital clinical laboratory. Other serum concentrations ( $\mathrm{Na}, \mathrm{K}$, bicarbonate, $\mathrm{Cl}$, glucose, Hct, and BUN) were determined using an Abbott i-Stat machine and EC8+ cartridges.

\section{Human Tissue Histology}

Surgical samples of normal human kidney and of acute tubular necrosis (ATN) were obtained from nephrectomy specimens with permission of Addenbrooke's Hospital ethical committee. Tissues $<1 \mathrm{~mm}$ thick was fixed in $4 \%$ formaldehyde in $0.1 \mathrm{~mol} / \mathrm{L}$ PIPES buffer $(\mathrm{pH} 7.5)$ for 1.5 hours at $4^{\circ} \mathrm{C}$ and embedded in paraffin-wax. For immunofluorescence studies, 5-micron thick sections were dewaxed in xylene and rehydrated in descending series of ethanol solutions before immunostaining. Antigen-retrieval was uslng $50 \mu \mathrm{g} / \mathrm{ml}$ Proteinase-K (Sigma) for 4 minutes at room temperature. Nonspecific antibody binding was blocked in blocking buffer containing 10\% fetal calf serum in $0.1 \mathrm{~mol} / \mathrm{L}$ Tris buffered saline $(\mathrm{pH} \mathrm{7.5}$; TBS/FCS) for 15 minutes at room temperature. Sections were then incubated overnight at $4{ }^{\circ} \mathrm{C}$ with goat antiNogo-B antibody (Santa Cruz) at 1:100 dilution in TBS/ FCS followed by 1:100 dilution in anti-goat AlexaFluor488 (Invitrogen) for 1 hour at room temperature. This was followed by 10 minutes incubation in Topro-3، iodide (Invitrogen) for nuclear detection. Sections were mounted in Vectashield mounting media (Vector Laboratories) and examined in Leica TCS/NT Confocal Laser Scanning Microscopy.

\section{Mouse Tissue Histology}

Kidneys were bisected, fixed overnight in 4\% PFA, embedded in paraffin, and sections cut at a thickness of 6 microns. For immunodetection of proteins, sections were rehydrated through sequential washes in xylene and graded alcohol solutions. When appropriate, endogenous peroxidase activity was blocked by incubation in $3 \% \mathrm{H}_{2} \mathrm{O}_{2}$ in methanol. Antigen retrieval was performed by boiling sections in citric acid solution for 15 minutes. Nonspecific antibody binding was blocked using blocking buffer (10\% donkey serum, 0.5\% BSA, 0.1\% Triton-X) for 30 minutes at room temperature. Primary antibody (or $\alpha$ Nogo anti-serum) was diluted in $10 \%$ donkey serum/ $0.5 \% \mathrm{BSA}$, and incubated overnight at $4^{\circ} \mathrm{C}$ (or in some cases at room temp for 2 hours). After washes in PBS, secondary antibody (Biotin-conjugated anti-rabbit (Jackson Immunoresearch) was diluted 1:200 in PBS and incubated for 1 hour at room temperature followed by streptavidin-linked peroxidase (Vectastain ABC kit, Vector Laboratories) and a colorimetric peroxidase substrate (No- 
vaRed, Vector Laboratories). For immunofluorescence, fluorochrome-conjugated secondary antibodies [anti-rabbit Cy5 (Jackson Immunoresearch), Alexafluor anti-rabbit 568 (Invitrogen)] were diluted 1:500 in PBS and incubated for 1 hour at room temperature. Lectins were diluted 1:100 to $\sim 20 \mu \mathrm{g} / \mathrm{ml}$ and incubated for 1 hour at room temperature. Sections were mounted in Vectashield hardset mounting media (Vector Laboratories) and analyzed by fluorescence microscopy using a Zeiss Axiovert fluorescence microscope or a Nikon Eclipse 80i light microscope. Picrosirius red staining was performed by the Yale Pathology Core Tissue Services. Quantitation of F4/80, or picrosirius red staining was performed by collecting 12 high-powered images from areas evenly distributed around the cortex of each section and subsequently analyzed with Image $\mathrm{J}$ software (http://rsbweb.nih.gov/ij).

\section{X-Gal Staining}

Tissue was fixed in $0.1 \%$ glutaraldehyde and $0.5 \%$ formaldehyde in PBS ( $\mathrm{pH}$ 7.4) and then stained either as whole mount, or first frozen in OCT compound, and cyrosectioned at a thickness of 8 microns. The stain solution [0.5 mg/ml X-gal (American Bioanalytical)/2.5 mmol/L Kferricyanide/2.5 mmol/L K-ferrocyanide/ $1 \mathrm{mmol} / \mathrm{L} \mathrm{MgCl} 2 /$ $0.01 \% N P-40]$ was applied at $37^{\circ} \mathrm{C}$ for approximately 50 minutes (whole kidneys) or overnight (sections). WT kidneys were always stained simultaneously to evaluate the presence of nonspecific staining unrelated to the recombinant lacZ gene. Under these conditions, minimal nonspecific staining was observed in both uninjured and injured WT kidneys.

\section{Pathological Scoring}

Hematoxylin and eosin-stained tissue sections were examined in a blinded fashion by a board certified renal pathologist (G.M.). Sections were scored on a four-point scale with 0 denoting no injury; 1 , mild; 2, moderate; and 3 , severe injury. Sections were evaluated separately for tubular atrophy and tubular necrosis.

\section{Western Blotting}

Proteins were extracted from tissues that were first frozen in liquid nitrogen and then pulverized by cooled mortar and pestle. Tissue was then incubated in extraction buffer [50 mmol/L Tris, pH 7.4/1\% NP-40/0.1\% SDS/0.1\% deoxycholic acid/0.1 mmol/L EDTA/0.1 mmol/L EGTA/2 $\mathrm{mg} / \mathrm{ml}$ protease cocktail (Roche)/ $1 \mathrm{mmol} / \mathrm{L}$ Pefabloc SC (Roche)] for 60 minutes at $4^{\circ} \mathrm{C}$. Equal amounts of protein were resolved by SDS-PAGE, followed by electrophoretic transfer onto 0.2-micron nitrocellulose membranes. Blots were blocked with casein (BioRad) in Tris-buffered saline, and then exposed to primary antibody diluted in casein solution (anti-Nogo 1:7500; anti- $\beta$ COP 1:1000; anti-AQP2 1:500) for either two hours at room temperature or overnight at $4^{\circ} \mathrm{C}$. Fluorophore-conjugated secondary antibodies (either 800 (Jackson Laboratories) or 680 (Invitrogen)) were diluted 1:5000 in Tris-buffered saline with $0.1 \%$ Tween-20 and incubated on the blot for 1 hour at room temperature. Bands were visualized using an Odyssey laser scanner (Li-cor Corp.) and further analyzed using Adobe Photoshop software (Adobe).

\section{Quantitative PCR}

Total RNA was extracted from frozen pulverized tissues using Trizol (Invitrogen) followed by further purification with RNEasy columns (Qiagen). Reverse transcription was performed on equal amounts of RNA using SuperScript II reverse transcriptase enzyme kit (Invitrogen) and a mix of random hexamer and oligo-dT primers. A BioRad iCycler machine was used with CyberGreen mastermix (BioRad) in conjunction with primer pairs designed to interrogate the genes of interest. Data were normalized to the expression level of $18 \mathrm{~S}$ RNA within each sample, and then expressed relative to the level in the WT contralateral unobstructed (CLU) kidney. Primers used were: 18S rRNA: 5'-TTCCGATAACGAACGAGACTCT-3' and 5'TGGCTGAACGCCACTTGTC-3'; NogoB1: 5'-CGGGCGCAGTGGTTGTTG-3' and 5'-CCGTTACACTGACAATGCTG-3'; RTN3 5'-AGAGTGGGATTTCTTCCGTTGGGT-3' and 5'-TTCTCAGGTGGCATTCTGGGAGTT-3'; MCP-1 5'-TTTCCACACTTCTATGCCTCCT-3' and 5'ATGGTCCTGAAGATCACAGCTT-3'; TNF $\alpha$ 5'-ACGGCATGGATCTCAAAGAC-3' and 5'-GTGGGTGAGGAGCACGTAGT-3'; Collagen $1(\alpha 1)$ 5'-AGAGCATGACCGATGGATTC-3' and 5'-CCTTCTTGAGGTTGCCAGTC3'; collagen $3(\alpha 1)$ 5'-CGTAAGCACTGGTGGACAGA-3' and 5'-CGGCTGGAAAGAAGTCTGAG-3'; $\alpha$ SMA: 5'TCCTCCCTGGAGAAGAGCTAC-3' and 5'- TATAGGTGGTTTCGTGGATGC-3'; TGF $\beta$ : 5' - TGCGCTTGCAGAGATTAAAA-3' and 5'-CTGCCGTACAACTCCAGTGA-3'.

\section{Statistical Analysis}

Quantitative data were expressed as mean \pm SE. For comparisons between groups the two-tailed Student's $t$-test function in Microsoft Excel was used, and values of $P<0.05$ were considered statistically significant.

\section{Results}

\section{Nogo Is Highly Expressed in the Renal Papilla}

We sought to identify cell types within the murine kidney that express the Nogo gene and protein using several different approaches. Initially, Nogo reporter mice engineered to express the lac $Z$ gene under the direction of the Nogo-A/B gene regulatory elements were used to examine cell specific gene expression in the kidney. ${ }^{19}$ Whole mount staining with $\mathrm{X}$-gal revealed intense expression in the papilla with scant positive cells elsewhere (Figure 1A). Frozen sections (Figure 1B) revealed intense staining of the inner medulla and papilla, similar to the whole mount, but also showed gene expression in certain tubular segments of the cortex. 
A

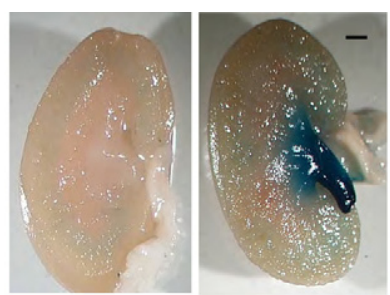

B

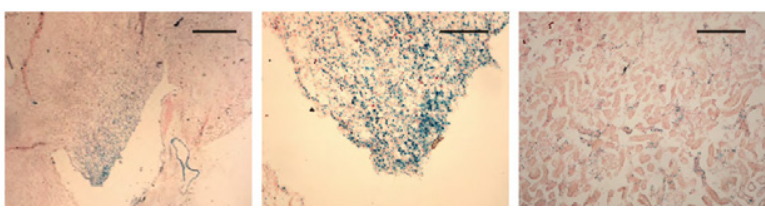

Figure 1. Nogo-A/B gene is expressed in distal nephron segments in murine kidneys. Nogo-A/B gene expression was assessed in Nogo lacZ reporter mice. Kidneys were fixed and developed with X-gal either as whole tissue (A) or after sectioning from frozen tissue blocks (B). In $\mathbf{A}$, the papilla and inner medulla stained heavily in the reporter kidney (right); there was no nonspecific staining in a WT kidney (left). In frozen sections (B), at low power (left), the predominance of staining was observed in the papilla and inner medulla. Scale bar $=200 \mu \mathrm{m}$. At higher magnification, intense staining was seen in the papilla (middle) and also in a subset of tubules in the cortex (right). Scale bar $=100 \mu \mathrm{m}$.

Next, we investigated Nogo-B protein expression by immunostaining of kidney sections with anti-Nogo antiserum. In immunofluorescence, immunohistochemistry, and Western blotting applications, this antisera does not significantly detect antigens in tissue from Nogo-A/B KO mice (Figure 2, A, B, and D). Immunostaining experiments on WT mice were consistent with X-gal data; most tubular epithelial cells in the inner medulla and papilla stained intensely, whereas only a subset of tubules in the cortex were significantly positive (Figure 2, A and B). Glomeruli were generally negative, but occasionally weak staining was observed. We used the lectins DBA and LTL to label distal and proximal nephron segments, respectively. DBA-positive tubules generally were also positive for Nogo-B, whereas LTL-positive tubules were never positive for Nogo-B (Figure 2C). These data suggest that the cortical tubules that are positive for Nogo-B are cortical segments of the distal nephron.

Next, we performed Western blotting on protein extracts from the cortex $(C)$ medulla $(M)$, and papilla $(P)$ of mouse kidneys (Figure 2D). Nogo-B was not detectable in protein homogenates from the whole kidney (not shown and Figure $3 \mathrm{C}$ ), likely because the molar ratio of Nogo-B to total protein in the homogenates was too low. Similarly, no Nogo-B was seen by Western in homogenates of cortex (C) or medulla (M) (Figure 2D). However, in extracts from the papilla, where nearly every tubule was positive for Nogo by immunohistochemistry and immunofluorescence (Figure 2, A and B), Nogo-B was consistently observed (but not Nogo-A, as judged by molecular weight). Generally, fractions which were positive for the collecting duct marker aquaporin-2 (AQP-2) were positive for Nogo-B, suggesting the highest concentration of the Nogo is in the collecting duct (Figure 2D). Of note, the Western blot data suggest that the expression of AQP-2 may be reduced in the Nogo-A/B KO mice. However, we
A
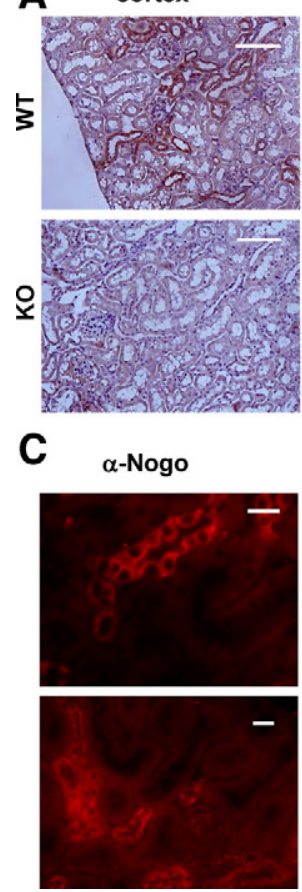

medulla
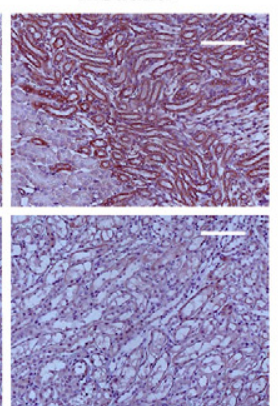

Lectin
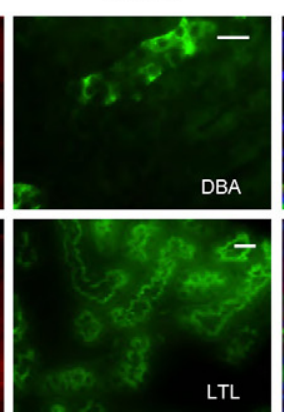

papilla

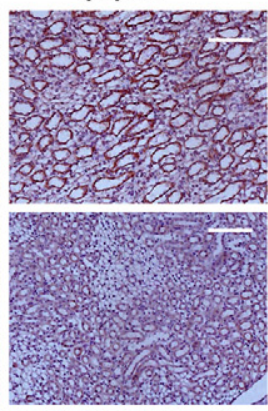

Merge

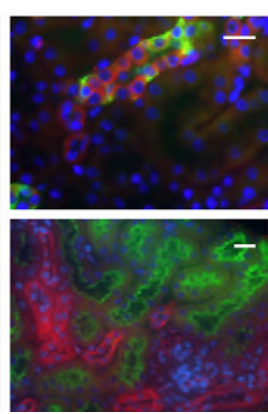

B
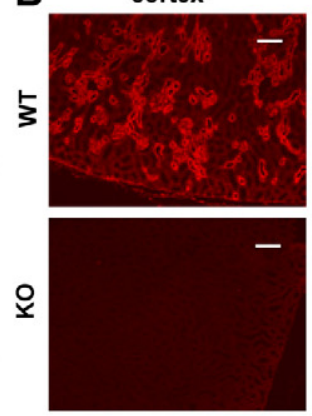

D

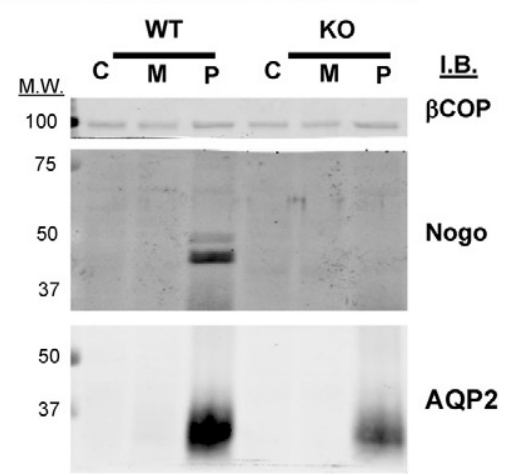

Figure 2. Nogo-B protein is expressed in distal nephron segments in murine kidneys. Anti-Nogo antiserum was used to perform immunohistochemistry (A) and immunofluorescence (B) on murine renal tissue sections. Specific Nogo staining was observed in the papilla and inner medulla, and also in distal nephron segments in the cortex. Minimal staining was seen in Nogo-A/B KO mice. Scale bars $=100 \mu \mathrm{m}$. C: Sections from WT kidneys were colabeled with anti-Nogo antiserum and fluorescein-conjugated DBA or LTL lectins. Whereas DBA-labeled tubules were also frequently positive for Nogo, (top row), LTL-labeled tubules (ie, proximal tubules) were never observed to stain positive for Nogo (bottom row). Scale bar $=20 \mu \mathrm{m}$. D: Western blot for Nogo. Kidneys from adult WT and Nogo-A/B KO were dissected into cortex (C), medulla (M), and papilla (P). Equal amounts of total extracted protein were resolved by SDS-PAGE and transferred to nitrocellulose. The blot was probed with anti- Nogo antiserum, antiaquaporin 2 (AQP2), a marker of the collecting duct, and anti- $\beta$-cotamer protein, a loading control $(\beta \mathrm{COP})$. Nogo-B was only detected in the papilla. None was detected in homogenates from Nogo-A/B KO animals. 
A

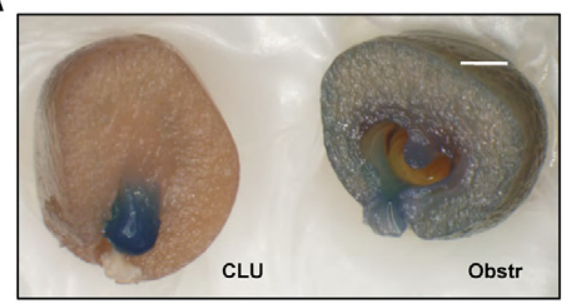

C

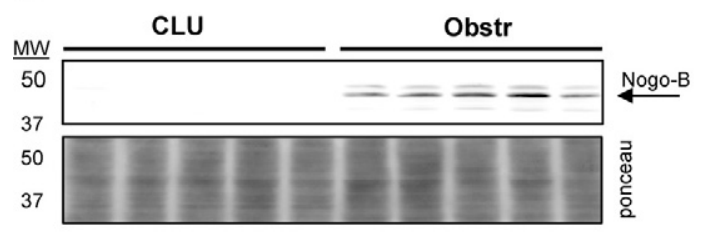

B

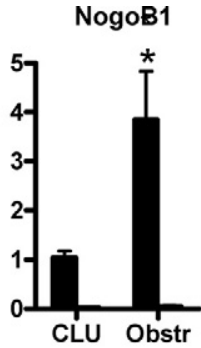

D

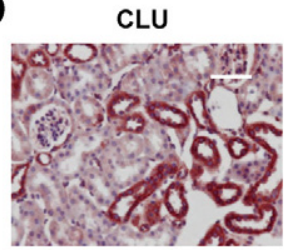

E

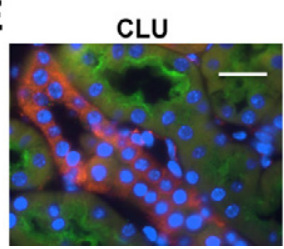

RTN3

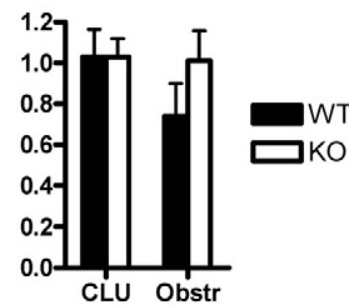

Obstr

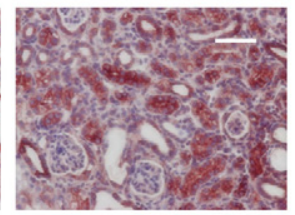

Obstr

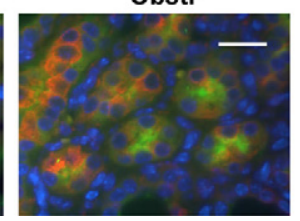

Figure 3. Changes in Nogo-B expression after UUO in the mouse kidney. A: UUO was performed on Nogo-A/B lacZ reporter mice. After 8 days, the mice were sacrificed, and the kidneys were fixed and stained with Xgal for 50 minutes at $37^{\circ} \mathrm{C}$. The obstr kidney showed significantly more Nogo gene expression than the CLU kidney. Results were identical in four additional mice. Scale bar $=\sim 1 \mathrm{~mm}$. B: Quantitative PCR analysis of Nogo-B1 and RTN3 mRNA levels in obstr and CLU kidneys after 8 days in WT and Nogo-A/B KO mice $(n=5)$. Although Nogo-B1 levels increased significantly, RNT3 levels were unchanged in both KO mice compared with WT, as well as in obstr relative to CLU kidneys. ${ }^{*} P<0.05$ relative to CLU. C: Top, Western blot for Nogo-B (arrow) in homogenates of CLU and obstr kidneys. Bottom, the blot was stained with Ponceau S to confirm equal loading of protein. Nogo-B was only detectable in obstr kidneys. D: Immunohistochemistry was performed on CLU and obstr kidneys after 8 days of UUO. The cortices showed more Nogo staining in the obstr (right) compared to the CLU (left) kidneys. Scale bar $=50 \mu \mathrm{m}$. E: To better characterize the expression of Nogo-B before and after injury, sections were colabeled with LTL (green) and anti-Nogo (orange). In the CLU kidney, LTL-positive proximal tubules did not simultaneously express Nogo. However, after 8 days of UUO, tubules that were positive for both LTL and Nogo-B were readily identified, suggesting that injury induces proximal tubules to express Nogo-B. Scale bar $=20 \mu \mathrm{m}$.

did not detect changes in urine osmolality in the NogoA/B KO mice (Table 1), so the physiological significance of this apparent reduction in AQP-2 is not clear. In sum, the results show that Nogo-B is expressed primarily in the epithelial cells of the distal portions of the nephron, especially the collecting duct.

Table 1. Metabolic Profile of WT and Nogo-A/B KO Mice

\begin{tabular}{lcc}
\hline \multicolumn{1}{c}{ Parameters } & \multicolumn{1}{c}{ WT } & \multicolumn{1}{c}{ KO } \\
\hline Weight $(\mathrm{g})$ & $24 \pm 2.2$ & $24 \pm 1.9$ \\
Na (mEq/L) & $145 \pm 0.7$ & $145 \pm 1.3$ \\
K (mEq/L) & $4.6 \pm 0.3$ & $4.5 \pm 0.1$ \\
Cl (mmol/L) & $111 \pm 1.1$ & $111 \pm 0.5$ \\
Bicarb (mmol/L) & $28.4 \pm 0.5$ & $28.0 \pm 1.4$ \\
Glucose (mg/dL) & $184 \pm 17$ & $178 \pm 24$ \\
Hct (\%) & $43.0 \pm 1.6$ & $42.0 \pm 2.6$ \\
BUN (mg/dL) & $20.8 \pm 2.9$ & $19.8 \pm 1.7$ \\
SCr $(\mathrm{mg} / \mathrm{dL})$ & $0.15 \pm 0.01$ & $0.16 \pm 0.01$ \\
Urine Osm* $(\mathrm{mOsm} / \mathrm{L})$ & $1960 \pm 606$ & $1975 \pm 336$ \\
\hline
\end{tabular}

Blood and urine were collected from adult age-matched WT and Nogo-A/B KO mice for analysis. Data are presented as the mean \pm SD. Serum creatinine (SCr) levels were determined by liquid chromatography at the Mouse Metabolic Phenotyping Center at Yale University. Urine osmolality values were determined in the Yale-New Haven Hospital clinical laboratory. All other values were determined using an Abbott i-Stat machine and EC8+ cartridges. $n=4-6$, except when indicated by an asterisk, $n=3-4$. No statistically significant differences were observed in the measured parameters between WT and KO mice.

\section{Alterations in Nogo Expression in a Model of Unilateral Ureteral Obstruction}

Published evidence suggests that endogenous Nogo-B may limit the extent of vascular injury, and vascular injury has been shown to alter levels of Nogo expression in vascular smooth muscle. ${ }^{5,10}$ We speculated that Nogo-B might play a similar role in models of renal injury. To test this hypothesis, we initially examined changes in gene expression in Nogo lacZ reporter mice after UUO for 8 days. In the obstructed (obstr) kidney, there was much more intense X-gal staining (reflecting enhanced Nogo gene expression) throughout the cortex than in the CLU kidney (Figure 3A). A concomitant decrease in Nogo gene expression in the papilla was observed, although the significance of this is uncertain because the architecture of the papilla was shrunken and distorted after UUO (Figure 3A). To confirm the changes in Nogo gene expression, quantitative PCR (qPCR) was performed on RNA extracted from whole kidneys. After obstruction, Nogo-B1 mRNA levels increased approximately fourfold (Figure $3 \mathrm{~B}$ ), however the levels of a related reticulon family member Rtn 3 were not elevated after obstruction (Figure 3B), suggesting that the changes were specific to Nogo-B and not to reticulons in general.

The changes in Nogo gene expression were mirrored at the level of protein as judged by Western blotting of 
A
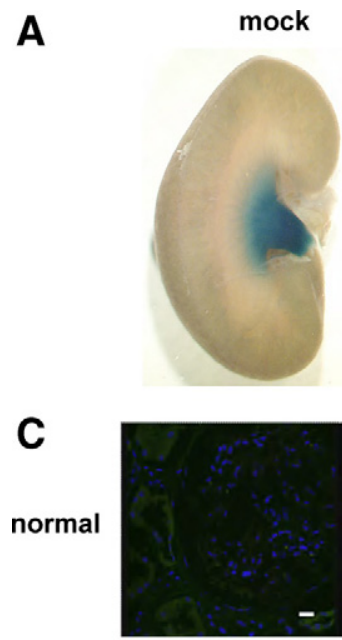

ATN

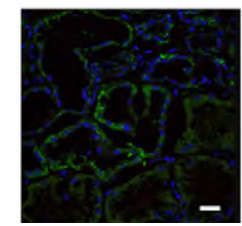

I/R
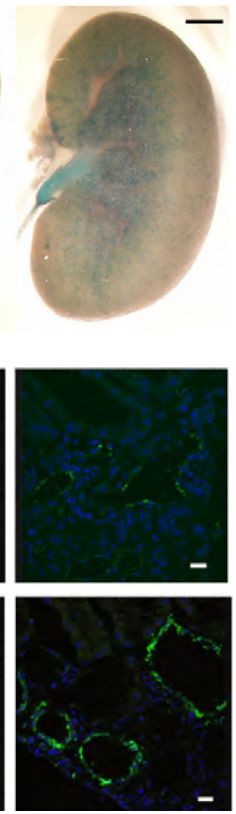

B
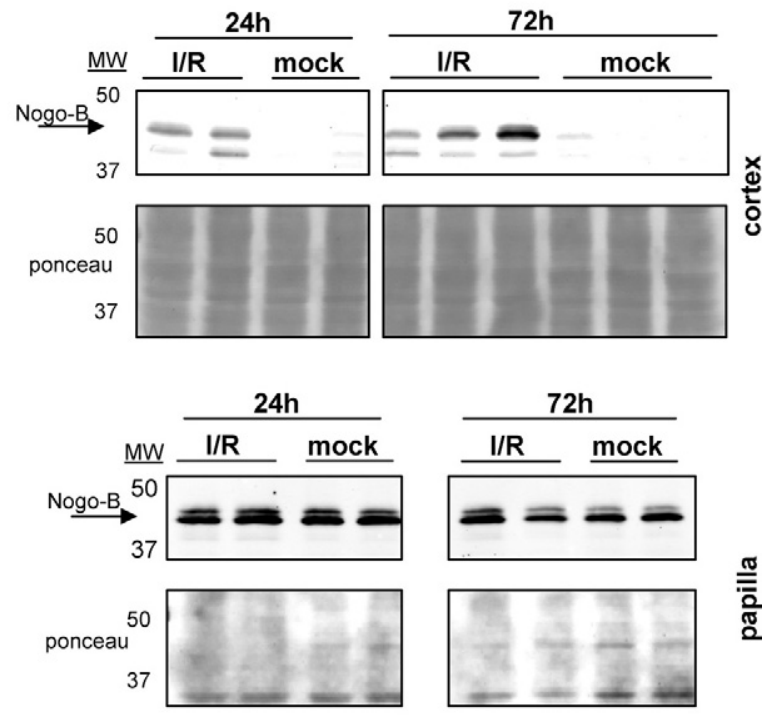

흘

Figure 4. Changes in Nogo expression after ischemic injury in the mouse kidney. A: Kidneys from Nogo lacZ reporter mice were stained with X-gal after either 30 minutes of ischemia and 72 hours of reperfusion or mock surgery. The injured kidney showed increased Nogo gene expression. Scale bar $=\sim 1$ mm. B: Western blot for Nogo-B was performed on protein extracts from papilla and cortices of mock treated kidneys and kidneys treated with 30 minutes ischemia and either 24 or 72 hours of reperfusion. Stains of blots with Ponceau S are shown to confirm equal loading. The Nogo-B band is indicated by arrow. Nogo-B was expressed in the papilla similarly in mock and I/R kidneys but was only observed in the cortices of kidneys subjected to I/R. C: Human biopsy specimens from normal and ATN kidneys were stained with the anti-Nogo antibody N18 (green). Increased Nogo expression was seen in the tubular epithelial cells of the ATN kidneys. Shown are representative images from the cortices of two normal and two ATN kidneys. Scale bar $=10 \mu \mathrm{m}$.

whole kidney homogenates. No Nogo-B was detected in CLU kidneys (as was the case in unoperated mice), whereas Nogo-B protein was observed in protein lysates from all five obstructed kidneys (Figure 3C). Similarly, immunohistochemistry of Nogo in obstr and CLU kidneys showed evidence of increased Nogo-B expression after injury (Figure 3D). In these sections, Nogo appeared to be primarily expressed in tubular epithelial cells, and not, for example, in infiltrating inflammatory cells.

To better characterize the expression pattern of Nogo after UUO, immunofluorescence experiments were performed in which sections were stained with anti-Nogo and the proximal tubule marker LTL. In CLU kidneys (as in unoperated mice), anti-Nogo (orange) and LTL (green) stained distinct populations of tubules (Figures 2C and 3E). In contrast, in obstructed kidneys, many tubules could be found to stain positive for both Nogo and LTL (Figure 3E), suggesting that after injury proximal tubules express Nogo-B. Five high-power fields were examined from the cortex of each of three CLU and three obstructed kidneys. In the CLU group, none of 52 examined LTL-positive tubules were positive for Nogo, whereas in the obstructed kidneys, 25 of 43 LTL-positive tubules were also positive for Nogo.

\section{Nogo-B Expression Is Altered in a Model of Renal Ischemia and Reperfusion}

To assess whether changes in Nogo-B expression levels were unique to UUO or a general feature of kidney injury, we performed unilateral ischemia/reperfusion (I/R) exper- iments. Whole mount staining of kidneys from Nogo lac $Z$ reporter mice revealed increased $X$-gal staining in the injured kidney compared with the contralateral mockoperated kidney after 72 hours of reperfusion (Figure 4A). Western blots of kidney fractions revealed that Nogo-B was consistently detectable in the cortex of kidneys after ischemia, but not in contralateral mock-operated controls (Figure 4B). These differences were apparent at both 24 and 72 hours of reperfusion. Nogo-B expression in the papilla was present in mock operated kidneys and was not affected by I/R as judged by Western blotting (Figure 4B), though gene expression may have been diminished as judged by $\mathrm{X}$-gal staining of reported mice kidneys (Figure 4A).

In an effort to detect whether similar changes in Nogo expression occur in humans after renal injury, immunofluorescence for Nogo was performed on human renal biopsy specimens. Samples from kidneys with ATN displayed more Nogo-B staining in tubular epithelial cells as compared with normal controls (Figure 4C). These results suggest that as in mice, human kidneys may up-regulate Nogo-B expression in response to renal injury.

\section{Examination of Renal Parameters in Nogo-A/B Knockout Mice}

Nogo-A/B KO mice were studied to assess the effects of the loss of Nogo on the kidney. At baseline, the loss of Nogo-A/B did not affect the gross or microscopic renal appearance (Figures 1A, 2A, and 5A). Analyses of blood and urine chemistries also demonstrated no significant 
A

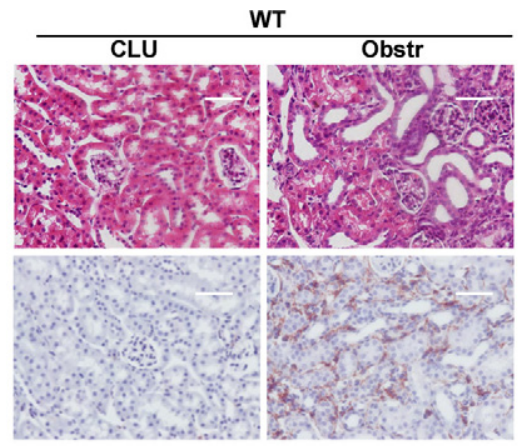

B

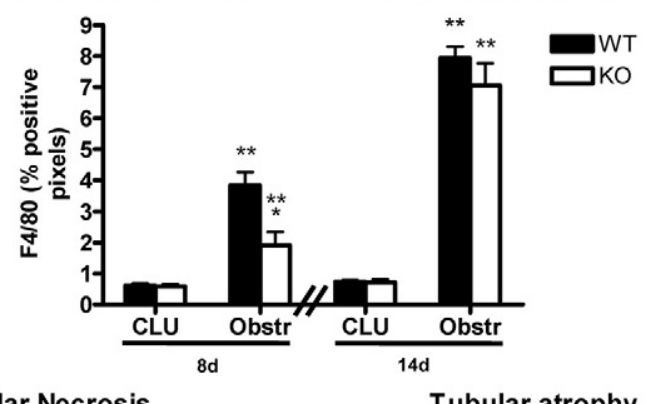

C

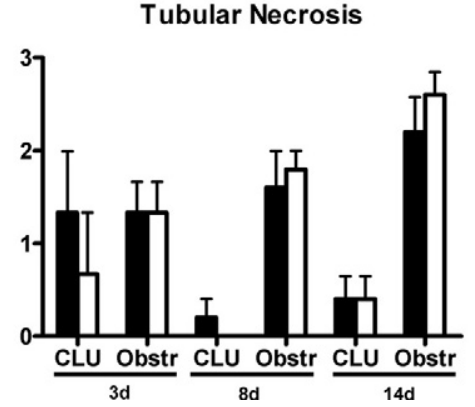

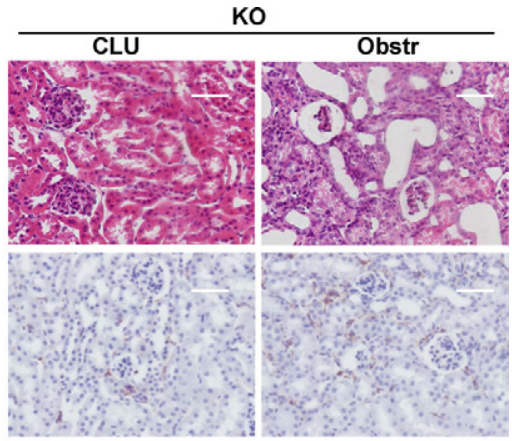

Tubular atrophy

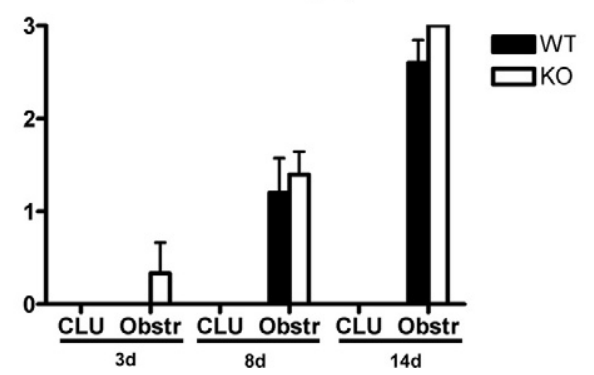

Figure 5. Characterization of histology and macrophage infiltration in Nogo-A/B KO kidneys in the UUO model. A: Top row, $\mathrm{H}+\mathrm{E}$ stained sections taken from either the CLU or the obstr kidneys 8 days after UUO. WT and KO CLU kidneys were indistinguishable. After UUO, both showed signs of injury, including dilated tubules. Scale bar $=50 \mu \mathrm{m}$. Bottom row, Representative images of macrophage infiltration after 8 days using immunohistochemistry with antiF4/80 antibody. Scale bar $=50 \mu \mathrm{m}$. B: Quantitation by digital image analysis of $\mathrm{F} 4 / 80$ positive staining. ${ }^{*} P<0.02$ compared to WT obstr; ${ }^{* *} P<$ 0.05 compared to corresponding CLU; $n=5$ in each group. C: H\&E-stained sections were examined by a renal pathologist in a blinded fashion and scored on a 4-point scale (with " 0 " denoting no injury and " 3 " denoting severe injury). Although the severity of injury increased from 3 to 14 days, there was no significant difference between WT and KO kidneys. $n=3$ at 3 days and $n=5$ at 8 and 14 days. differences (Table 1), suggesting that basic renal function was essentially normal in Nogo-A/B KO mice. Given the marked changes in Nogo-B expression that were observed after UUO, we sought to determine whether WT and Nogo-A/B KO mice displayed differences in this model. Because macrophage infiltration is a major event in the pathophysiology of ureteral obstruction, ${ }^{20,21}$ and we have observed changes in leukocyte recruitment to sites of various types of tissue injury in Nogo-A/B KO mice, ${ }^{22}$ we assessed whether there were any defects in macrophage recruitment after UUO in Nogo-A/B KO kidneys. There was a significant delay in macrophage recruitment/retention in the obstructed kidney in Nogo-A/B $\mathrm{KO}$ mice as compared with WT mice (Figure 5). In particular, after 8 days of UUO, macrophage recruitment was reduced by approximately 50\%. However, by 14 days, the total number of F4/80-positive cells was not different (Figure 5B), suggesting that other factors had compensated for the defect in macrophage recruitment.

This delay in macrophage recruitment did not translate into obvious reductions in renal injury. Independent assessment of the extent of renal injury (in a blinded fashion by a renal pathologist on a scale of $0-3$ to delineate the levels of tubular necrosis and tubular atrophy) in mice after UUO from 3 to 14 days revealed no significant differences between WT and Nogo-A/B KO mice (Figure $5 \mathrm{C}$ ). In addition the levels of renal fibrosis (as determined by picrosirius red staining and digital image analysis after 14 days) and of expression of inflammatory and profibrotic genes (as determined by quantitative PCR after 8 days) were not significantly different between the strains (Figure 6, A-C).

\section{Discussion}

The central findings of this article study that in mice Nogo-B is highly expressed in the epithelial cells of the distal segments of the nephron and that in two different injury models (UUO and I/R), and in ATN in humans, the expression pattern of Nogo-B changes dramatically (Figures 3 and 4). In particular, after UUO, cortical Nogo-B expression is increased, driven largely by new expression in proximal tubular epithelia (Figure 3E). These conclusions are supported by multiple lines of data, including X-gal staining of reporter mice, quantitative PCR, and immunostaining and Western blotting of dissected kidneys. The baseline expression of Nogo-B in the collecting duct is supported by a proteomics study in which Nogo-B was identified in preparations from the rat inner medullary collecting duct. ${ }^{23}$

The injury-induced up-regulation of Nogo-B is not a general response among reticulons, as mRNA levels of another reticulon (Rtn 3 ) were not affected by either ge- 

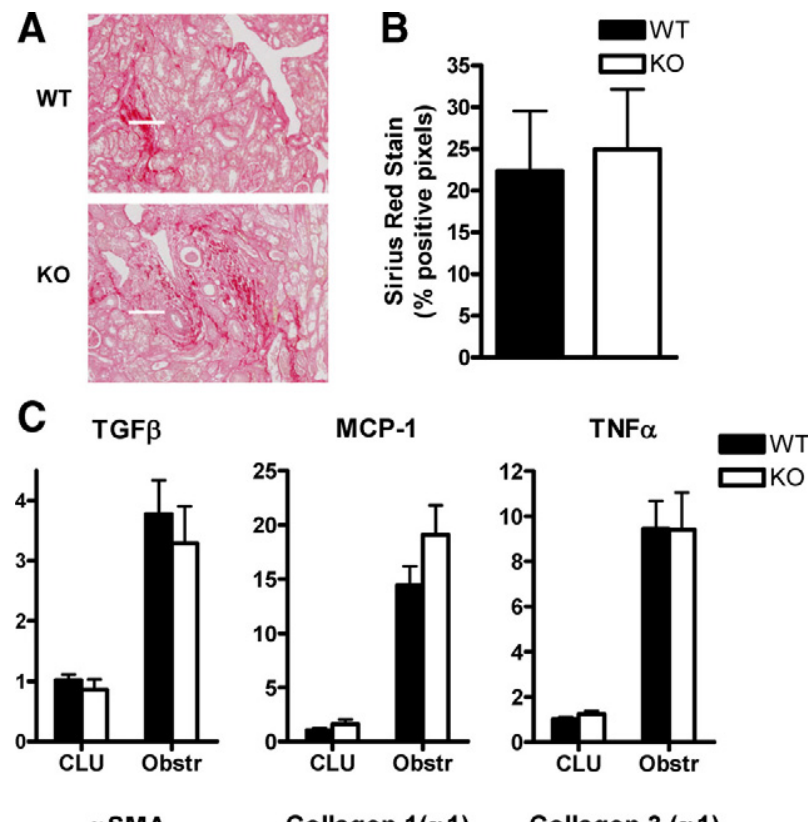

$\alpha$ SMA
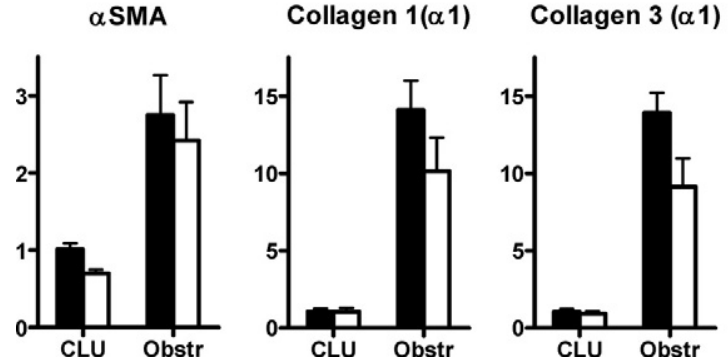

Figure 6. Characterization of fibrosis and inflammation in obstructed kidneys after UUO. A: Kidneys were stained with picrosirius red to evaluate the extent of collagen deposition; representative images are shown. Scale bar $=$ $50 \mu \mathrm{m}$. B: Quantification of staining by digital image analysis. There were no significant differences between WT and Nogo-A/B KO mice ( $n=5$ each) Similar analyses were undertaken after 8 days; although much less fibrosis had accumulated at this time point, no significant differences between WT and KO were noted (data not shown). C: Quantitative PCR analysis of gene expression in WT and Nogo-A/B KO kidneys after 8 days of UUO. qPCR was performed using primers specific for the indicated genes (TGF $\beta$, transforming growth factor $\beta$; MCP-1, monocyte chemoattractant protein 1 ; TNF $\alpha$, tumor necrosis factor $\alpha$; SMA, $\alpha$ smooth muscle actin). For all genes tested and in both genotypes, $P<0.05$ for obstructed compared with CLU kidneys. In no case of obstructed kidneys was $P<0.05$ for WT compared with Nogo-A/B KO. $n=5$ per group.

netic deletion of Nogo-B or by UUO (Figure 3B). It is not clear what the stimulus for increased Nogo-B expression is. Two clues are that i) two distinct injury modalities each led to similar changes in Nogo-B expression; and ii) increased Nogo-B expression occurred within 24 hours in the I/R model (Figure 4B). These observations suggest that the stimulus is not unique to either injury but instead is a common early feature of both models such as cytokine release or tissue hypoxia.

The large change in Nogo-B expression after renal injury suggests that immunostaining for Nogo-B may ultimately aid in the pathological analysis of renal tissue. Future studies will be needed to better define the sensitivity and specificity of Nogo-B expression as a marker of renal injury in humans. In addition, analysis of Nogo in the serum or urine may also be of potential diagnostic use; elevations of Nogo serum levels were found in a biomarker-discovery study of dilated cardiomyopathy. ${ }^{11}$ But to our knowledge, published studies of urine proteomics designed to identify novel biomarkers for renal injury have not identified Nogo to date. Efforts by us to study Nogo levels in mice with and without renal injuries have been hampered by the inability of the ELISA developed in our laboratory to detect the murine isoform of Nogo. However, future studies of Nogo-B levels human urine in the setting of renal injury are planned.

Interestingly, despite evidence that Nogo-B expression is tightly regulated both at baseline and after renal injury, the phenotype of the Nogo-A/B knockout mouse was very subtle. The only notable difference between WT and $\mathrm{KO}$ mice was a delay in the recruitment of macrophages to the kidney after UUO in KO animals. It is not clear whether the decrease in macrophage recruitment is due to the absence of Nogo-B in the renal epithelium, or in the macrophage itself, where Nogo-B is expressed. ${ }^{22,24}$ Other indices of the injury response (such as histological changes, fibrosis, and expression of proinflammatory and profibrotic genes) were not significantly different between WT and Nogo-A/B KO mice (Figures $5 \mathrm{C}$ and 6 ). Possible explanations for this apparent contradiction are that the delay in macrophage recruitment is too transitory to be significant in the overall elaboration of injury, that other compensatory inflammatory stimuli obscure the function of Nogo-B when it is absent, or that the polarization of macrophages in the Nogo-A/B $\mathrm{KO}$ animals is different from that in the WT such that a similar amount of tissue injury and fibrosis develops despite overall reduced numbers of macrophages. Regarding the latter point, emerging data suggest that all macrophages are not alike, and that some subsets are profibrotic whereas others serve to limit the extent of injury. ${ }^{25-27}$

Of note, our results are similar to those reported recently in a mouse model of Alport's disease. ${ }^{28}$ In that study, blockade of MCP-1 reduced the infiltration of macrophages into both the glomeruli and the interstitium but did not improve glomerular or interstitial pathology or overall survival. Furthermore, the transient nature of the alteration in inflammation reported here in the Nogo-A/B $\mathrm{KO}$ mice is not unusual in the context of prior studies of gene deletions in UUO, where effects that were apparent at early time points frequently became statistically insignificant at later time points. ${ }^{29-33}$ For example, in plasminogen activator inhibitor-1-deficient mice, interstitial macrophages are reduced relative to WT mice at 3 and 7 days of UUO, but by 14 days the difference is no longer statistically significant. ${ }^{33}$ Presumably this phenomenon reflects the multiplicity of redundant signaling pathways activated by UUO.

The interesting regulation of Nogo-B expression in the kidney before and after injury suggests not only that it may serve as a useful pathological biomarker of injury, but that it may also play a role in the genesis or resolution of renal injury. Future studies of different injury conditions, perhaps guided by emerging insights into the molecular roles of Nogo-B, will likely clarify its physiological function in the kidney as well as its characteristics as a marker of tissue injury. 


\section{Acknowledgments}

We acknowledge the technical support of Gunilla Thulin, Zhengrong Hao, and Roger Babbitt as well as the administrative assistance of Carol Muzzey and Diane Strumpf. Dr. Marin also thanks Drs. Peter Aronson, Jordan Pober, Lloyd Cantley, Michael Caplan, and Michael Kashgarian for helpful advice.

\section{References}

1. De Craene JO, Coleman J, Estrada de Martin P, Pypaert M, Anderson S, Yates JR, 3rd, Ferro-Novick S, Novick P: Rtn1p is involved in structuring the cortical endoplasmic reticulum. Mol Biol Cell 2006, 17:3009-3020

2. Voeltz GK, Prinz WA, Shibata Y, Rist JM, Rapoport TA: A class of membrane proteins shaping the tubular endoplasmic reticulum. Cell 2006, 124:573-586

3. Liu Y, Vidensky S, Ruggiero AM, Maier S, Sitte HH, Rothstein JD: Reticulon $\mathrm{RTN} 2 \mathrm{~B}$ regulates trafficking and function of neuronal glutamate transporter EAAC1. J Biol Chem 2008, 283:6561-6571

4. Wakana Y, Koyama S, Nakajima K-i, Hatsuzawa K, Nagahama M, Tani K, Hauri H-P, Melançon P, Tagaya M: Reticulon 3 is involved in membrane trafficking between the endoplasmic reticulum and Golgi. Biochem Biophys Res Commun 2005, 334:1198-1205

5. Acevedo L, Yu J, Erdjument-Bromage H, Miao RQ, Kim JE, Fulton D, Tempst P, Strittmatter SM, Sessa WC: A new role for Nogo as a regulator of vascular remodeling. Nat Med 2004, 10:382-388

6. Miao RQ, Gao Y, Harrison KD, Prendergast J, Acevedo LM, Yu J, Hu F, Strittmatter SM, Sessa WC: Identification of a receptor necessary for Nogo-B stimulated chemotaxis and morphogenesis of endothelial cells. Proc Natl Acad Sci USA 2006, 103:10997-11002

7. Kuang E, Wan Q, Li X, Xu H, Zou T, Qi Y: ER stress triggers apoptosis induced by Nogo-B/ASY overexpression. Exp Cell Res 2006, 312:1983-1988

8. Chen MS, Huber AB, van der Haar ME, Frank M, Schnell L, Spillmann AA, Christ F, Schwab ME: Nogo-A is a myelin-associated neurite outgrowth inhibitor and an antigen for monoclonal antibody $\mathbf{I N}-1$. Nature 2000, 403:434-439

9. GrandPre T, Nakamura F, Vartanian T, Strittmatter SM: Identification of the Nogo inhibitor of axon regeneration as a Reticulon protein. Nature 2000, 403:439-444

10. Kritz AB, Yu J, Wright PL, Wan S, George SJ, Halliday C, Kang N, Sessa WC, Baker AH: In vivo modulation of Nogo-B attenuates neointima formation. Mol Ther 2008:1798-1604

11. Gramolini AO, Kislinger T, Alikhani-Koopaei R, Fong V, Thompson NJ, Isserlin R, Sharma P, Oudit GY, Trivieri MG, Fagan A, Kannan A, Higgins DG, Huedig H, Hess G, Arab S, Seidman JG, Seidman CE, Frey B, Perry M, Backx PH, Liu PP, MacLennan DH, Emili A: Comparative proteomics profiling of a phospholamban mutant mouse model of dilated cardiomyopathy reveals progressive intracellular stress responses. Mol Cell Proteomics 2008, 7:519-533

12. Bullard TA, Protack TL, Aguilar F, Bagwe S, Massey HT, Blaxall BC: Identification of Nogo as a novel indicator of heart failure. Physiol Genomics 2008, 32:182-189

13. Lee WS, Kim SW, Hong SA, Lee TJ, Park ES, Kim HJ, Lee KJ, Kim TH, Kim CJ, Ryu WS: Atherosclerotic progression attenuates the expression of Nogo-B in autopsied coronary artery: pathology and virtual histology intravascular ultrasound analysis. J Korean Med Sci 2009 $24: 596-604$
14. Rodriguez-Feo JA, Hellings WE, Verhoeven BA, Moll FL, de Kleijn DP, Prendergast J, Gao Y, van der Graaf Y, Tellides G, Sessa WC, Pasterkamp G: Low levels of Nogo-B in human carotid atherosclerotic plaques are associated with an atheromatous phenotype, restenosis, and stenosis severity. Arterioscler Thromb Vasc Biol 2007, 27: 1354-1360

15. Pan JW, Wei M, Yang PY, Zheng X, Li J, Lu Z, Zhao XX, Wu H, Kang $H$, Rui Y: Regulation of Nogo-B expression in the lesion of aortic aneurysms. Clin Exp Pharmacol Physiol 2007, 34:856-860

16. Huber AB, Weinmann O, Brosamle C, Oertle T, Schwab ME: Patterns of Nogo mRNA and protein expression in the developing and adult rat and after CNS lesions. J Neurosci 2002, 22:3553-3567

17. Fournier AE, GrandPre T, Strittmatter SM: Identification of a receptor mediating Nogo-66 inhibition of axonal regeneration. Nature 2001 , 409:341-346

18. Zheng B, Ho C, Li S, Keirstead H, Steward O, Tessier-Lavigne M: Lack of enhanced spinal regeneration in Nogo-deficient mice. Neuron 2003, 38:213-224

19. Kim J-E, Li S, GrandPre T, Qiu D, Strittmatter SM: Axon regeneration in young adult mice lacking Nogo-A/B. Neuron 2003, 38:187-199

20. Diamond JR: Macrophages and progressive renal disease in experimental hydronephrosis. Am J Kidney Dis 1995, 26:133-140

21. Henderson NC, Mackinnon AC, Farnworth SL, Kipari T, Haslett C, Iredale JP, Liu FT, Hughes J, Sethi T: Galectin-3 expression and secretion links macrophages to the promotion of renal fibrosis. Am J Pathol 2008, 172:288-298

22. Yu J, Fernández-Hernando $C$, Suarez $Y$, Schleicher M, Hao Z, Wright PL, Kyriakides TR, Sessa WC: Reticulon 4B (Nogo-B) is necessary for macrophage infiltration and tissue repair. Proc Natl Acad Sci USA 2009, 106:17511-17516

23. Barile M, Pisitkun T, Yu MJ, Chou CL, Verbalis MJ, Shen RF, Knepper MA: Large-scale protein identification in intracellular aquaporin-2 vesicles from renal inner medullary collecting duct. mol cell proteomics. 2005, 4:1095-1106

24. Rousseau S, Peggie M, Campbell DG, Nebreda AR, Cohen P: Nogo-B is a new physiological substrate for MAPKAP-K2. Biochem J 2005, 391:433-440

25. Mantovani A, Sica A, Locati M: Macrophage polarization comes of age. Immunity 2005, 23:344-346

26. Nishida M, Hamaoka K: Macrophage phenotype and renal fibrosis in obstructive nephropathy. Nephron Exp Nephrol 2008, 110:e31-36

27. Nishida M, Okumura Y, Fujimoto S, Shiraishi I, Itoi T, Hamaoka K Adoptive transfer of macrophages ameliorates renal fibrosis in mice. Biochem Biophys Res Commun 2005, 332:11-16

28. Clauss S, Gross O, Kulkarni O, Avila-Ferrufino A, Radomska E Segerer S, Eulberg D, Klussmann S, Anders HJ: Ccl2/Mcp-1 blockade reduces glomerular and interstitial macrophages but does not ameliorate renal pathology in collagen4A3-deficient mice with autosomal recessive Alport nephropathy. J Pathol 2009, 218:40-47

29. Chevalier RL, Forbes MS, Thornhill BA: Ureteral obstruction as a model of renal interstitial fibrosis and obstructive nephropathy. Kidney Int 2009, 75:1145-1152

30. Klahr S, Morrissey J: Obstructive nephropathy and renal fibrosis. Am J Physiol Renal Physiol 2002, 283:F861-F875

31. Bascands JL, Schanstra JP: Obstructive nephropathy: insights from genetically engineered animals. Kidney Int 2005, 68:925-937

32. Satoh M, Kashihara N, Yamasaki Y, Maruyama K, Okamoto K, Maeshima Y, Sugiyama H, Sugaya T, Murakami K, Makino H: Renal interstitial fibrosis is reduced in angiotensin $\|$ type 1a receptordeficient mice. J Am Soc Nephrol 2001, 12:317-325

33. Oda T, Jung YO, Kim HS, Cai X, Lopez-Guisa JM, Ikeda Y, Eddy AA: PAl-1 deficiency attenuates the fibrogenic response to ureteral obstruction. Kidney Int 2001, 60:587-596 